\title{
Frecuencia de factores relacionados con sepsis neonatal
}

\author{
Patricia J. Ostia-Garza* y Berenice Salzar-Espino \\ Servicio de Neonatología, Hospital Materno Perinatal Mónica Pretelini Sáenz, Toluca de Lerdo, Estado de México, México
}

\section{Resumen}

La sepsis neonatal es una situación clínica derivada de la invasión y proliferación de bacterias, hongos o virus en el recién nacido, que se manifiesta en los primeros 28 días de vida. La etiología es fundamentalmente bacteriana, fúngica y vírica en menos del $1 \%$ de los casos. Las bacterias más frecuentemente implicadas son Streptococcus agalactiae o Streptococcus del grupo B y Escherichia coli. Objetivo: Determinar la frecuencia de factores relacionados con sepsis neonatal en el Servicio de Neonatología del Hospital Materno Perinatal Mónica Pretelini Sáenz en el periodo del año 2013 al 2015. Metodología: Estudio descriptivo, transversal, retrospectivo y observacional, 378 expedientes estudiados (expedientes con diagnóstico de sepsis neonatal con germen aislado). Conclusión: Los factores relacionados con sepsis en el periodo neonatal fueron el uso de nutrición parenteral, la asistencia con ventilación mecánica y la utilización de vías centrales. Los microorganismos más frecuentemente aislados fueron E. coli (21.5\%) y Klebsiella pneumoniae (20.5\%).

Palabras clave: Sepsis neonatal. Prematurez. Factores de riesgo.

\section{Frequency of risk factors associated with neonatal sepsis}

\section{Abstract}

Neonatal sepsis is that clinical situation derived from the invasion and proliferation of bacteria, fungi or viruses in the newborn, which manifests itself in the first 28 days of life. The etiology is fundamentally bacterial, since sepsis due to fungi and viruses accounts for less than $1 \%$ of cases. Among the bacteria most frequently involved are Streptococcus agalactiae or Streptococcus group B and Escherichia coli. Objective: To determine the frequency and factors related to neonatal sepsis in the neonatology service of the Mónica Pretelini Sáenz Perinatal Maternity Hospital in the period from 2013 to 2015. Methodology: A descriptive, cross-sectional, retrospective and observational study was carried out, where all the records of newborns with diagnosis of neonatal sepsis with isolated germ were taken, with a total of 378 records, Conclusion: The factors related to neonatal sepsis in the period we're the use of parenteral nutrition, assistance with mechanical ventilation and use of central veins. The most frequently isolated microorganisms were Escherichia coli (21.5\%) and Klebsiella pneumoniae (20.5\%).

Key words: Neonatal sepsis. Preterm. Risk factors.

\section{Correspondencia:}

*Patricia J. Ostia-Garza

E-mail: patty_ostia@ hotmail.com
Fecha de recepción: 16-07-2019

Fecha de aceptación: 16-08-2021

DOI: 10.24875/PER.19000059
Disponible en internet: 28-12-2021

Perinatol Reprod Hum. 2021;35(1):3-9

www.perinatologia.mx 0187-5337/@ 2021. Instituto Nacional de Perinatología Isidro Espinosa de los Reyes. Publicado por Permanyer. Este es un artículo open access bajo la licencia CC BY-NC-ND (http://creativecommons.org/licenses/by-nc-nd/4.0/). 


\section{Introducción}

Las causas que explican las tres cuartas partes de la mortalidad neonatal en el mundo son: partos prematuros $(29 \%)$, asfixia $(23 \%)$ y sepsis $(25 \%)$. En zonas como América Latina, la incidencia de sepsis es alta (2.2 a 8.6 por cada 1,000 recién nacidos vivos). El $49 \%$ de todas las infecciones ocurre en menores de un año de edad y la mitad de estas durante el periodo neonatal'.

Las infecciones en el periodo neonatal con frecuencia son fatales y causan morbilidad significativa a largo plazo en los supervivientes si no se tratan de manera apropiada y rápida. El riesgo de transmisión de infecciones de madres embarazadas a sus hijos y de exposiciones en las primeras semanas de vida se ha reconocido durante siglos ${ }^{2}$. Se empezó a comprender que muchas de las enfermedades neonatales eran causadas por infecciones en la década de 1800. Sin embargo, no ha sido sino hasta los avances técnicos en la microbiología de los últimos 50 años cuando se ha apreciado realmente la magnitud de este fenómeno. En EE.UU., antes de la década de 1950 el estreptococo betahemolítico del grupo A fue el microorganismo patógeno predominante como causa de sepsis neonatal en los primeros días de vida. En relación con las mejoras prácticas de cuidado obstétrico la infección por esta entidad se hizo menos frecuente, por lo que Staphylococcus aureus y Escherichia coli se convirtieron en los microorganismos aislados con mayor frecuen$\mathrm{cia}^{2}$. El reconocimiento de los factores de riesgo de adquisición para estas bacterias llevó a cambios en las medidas de antisepsia en las salas de parto y neonatales. A principios de la década de 1970 el estreptococo betahemolítico del grupo $B$ se convirtió en la bacteria predominante en EE.UU. en las salas de parto, por lo que se desarrollaron programas de detección durante el embarazo ${ }^{2}$. Durante las dos últimas décadas, la infección más frecuente como causa de sepsis neonatal ha sido aquella por estafilococo coagulasa negativo, relacionada con catéteres venosos centrales 0 arteriales infectados. No obstante, el escenario está cambiando de nuevo como resultado de las estrategias de prevención de las infecciones relacionadas con catéter. Las preocupaciones ahora se centran en infecciones bacterianas que son abiertamente resistentes a los antibióticos de uso frecuente, como $S$. aureus resistente a meticilina, enterococos resistentes a vancomicina $o$ aquellos en los que se puede inducir resistencia con la exposición de muchos antibióticos como las bacterias gramnegativas productoras de betalactamasa de espectro extendido o de carbapenemasa. El surgimiento y la diseminación de estas bacterias han hecho considerablemente más complejo el tratamiento empírico de la sepsis en los neonatos. Son el grupo de pacientes donde no hay población bacteriana residente en su piel o sus superficies mucosas. La colonización se inicia con rapidez en las primeras horas de vida, pero el establecimiento de la flora bacteriana residente permanente requiere varios meses. Los neonatos presentan una deficiencia inmunitaria relativa en comparación con los lactantes de más edad y adultos. Los neonatos son casi exclusivamente dependientes de la fase innata del sistema inmunitario por la defensa inicial ante las infecciones, ya que no muestran inmunidad adaptativa previa ${ }^{2,3}$.

En relación con la incidencia de infecciones relacionadas con la atención sanitaria en los neonatos atendidos en unidad de cuidados intensivos neonatales, el riesgo es mayor con la ruptura prolongada de membranas, corioamnionitis materna, enfermedad febril materna, ruptura prematura de membranas, colonización materna por microorganismos maternos, prematurez, peso bajo al nacimiento, hospitalización materna o del neonato por tiempo prolongado, necesidad de procedimientos invasivos como intubación orotraqueal, colocación de catéteres centrales, intervenciones quirúrgicas, etc. La sepsis neonatal es aquella situación clínica derivada de la invasión y proliferación de bacterias, hongos o virus en el torrente sanguíneo del recién nacido y que se manifiesta en los primeros 28 días de vida ${ }^{3}$.

La presencia de enfermedad clínica distingue sepsis de una bacteriemia aislada. Los microorganismos que causan sepsis en el periodo neonatal varían con base en la región geográfica, la edad de gestación y estos factores relacionados con las poblaciones locales y la unidad de cuidados intensivos neonatales. Es indispensable el conocimiento de los microorganismos más probables que pueden causar infección en el área local para asegurar que se provea tratamiento antimicrobiano adecuado mientras se espera resultado de los cultivos y otras pruebas diagnósticas ${ }^{4}$. Los signos de sepsis pueden ser sutiles e incluyen inestabilidad de la temperatura, letargo, apnea o aumento de estas, incremento de la necesidad de soporte respiratorio, hipotonía y alimentación deficiente. La hipotermia es más frecuente que la hipertermia. Los signos adicionales incluyen taquipnea, cianosis, vómito y diarrea, se pueden observar también heces con positividad al grupo hemo y distensión abdominal, y en realidad no indican la fuente de infección. En algunos pacientes se 
observa hepatoesplenomegalia, ictericia, petequias, y por lo general no en el momento del cuadro clínico inicial. A veces se encuentran índices leucocitarios anormales, acidosis metabólica e hiperglucemia. Los neonatos con una cuenta baja de neutrófilos en el momento del cuadro clínico de sepsis pueden tener un peor resultado ${ }^{4}$. En nuestro país la información es limitada, aunque se reporta en el Instituto Nacional de Perinatología una incidencia de sepsis neonatal de 19 por cada 1,000 recién nacidos vivos. En el instituto Nacional de Pediatría Klebsiella pneumoniae constituye el microorganismo mayormente encontrado compatible con lo encontrado en otras instituciones mexicanas ${ }^{5}$. Sin embargo, el estreptococo betahemolítico del grupo B constituye en EE.UU. y Europa el agente etiológico más frecuente, mientras que en países menos industrializados los microorganismos gramnegativos siguen siendo los predominantes ${ }^{6}$. Se define como sepsis de inicio precoz o temprano a la que inicia en los primeros tres a cinco días y sepsis de inicio tardío a la que lo hace con sintomatología pasada la semana de vida ${ }^{6}$. Los neonatos prematuros tienen mayor riesgo de muerte que los de término. La cifra de leucocitos puede mostrar neutropenia, con un número relativamente grande de células inmaduras, o elevación del número de neutrófilos o ser normal. Puede haber trombocitopenia relacionada. El diagnóstico se hace por detección del microorganismo en hemocultivo, líquido cefalorraquídeo u otros cultivos del sitio infectado. La profilaxis materna puede obstaculizar la detección del microorganismo por cultivo de líquido cefalorraquídeo y hemocultivo ${ }^{7}$. Los catéteres vasculares son considerados indispensables en la atención de pacientes hospitalizados en la unidad de cuidados intensivos neonatales, sin embargo, esos factores cambian de acuerdo con el tipo de pacientes tratados en cada unidad y las características sociodemográficas de cada país y de cada región, e incluso de cada unidad ${ }^{8}$. En EE.UU. se encontró que la permanencia del catéter umbilical en un tiempo igual o superior a siete días (razón de momios [RM]: 2.5; intervalo de confianza del 95\% [IC 95\%]: 1.3-5-1) y la permanencia de catéter arterial un tiempo igual o superior a siete días (RM: 2.1; IC 95\%: 1.1-4.1) son factores de riesgo para infección nosocomial ${ }^{9}$.

En Canadá, Aziz, et al. encontraron que los factores de riesgo para infección nosocomial en pacientes menores de 1,500 gramos fueron edad gestacional entre 24 y 28 semanas (RM: 2.2; IC 95\%: 1.2-4.1), el uso de ventilación mecánica asistida (RM: 1.5; IC 95\%: 1.1-2.0) y el uso de nutrición parenteral (RM: 3.9; IC 95\%:
3.0-5.2), mientras que en pacientes mayores de 1,500 gramos fueron edad gestacional entre 29 y 32 semanas (RM: 1.9; IC 95\%: 1.3-2.6), presencia de una línea intravenosa periférica (RM: 1.9; IC 95\%: 1.1-3.2), el uso de catéter venoso central (RM: 1.6; IC 95\%: 1.2-2.2), la ventilación mecánica (RM: 2.9; IC 95\%: 1.6-5.0) y anomalías congénitas (RM: 2.9; IC 95\%: 2.2-3.8) ${ }^{10}$. En México, en un estudio realizado en 21 hospitales públicos, se encontró que hay cuatro factores independientes asociados al desarrollo de infección nosocomial: exposición a catéteres intravenosos (RM: 3.3; IC 95\%: 1.95.9), alimentación parenteral (RM: 2.1; IC 95\%: 1.0-4.5), ventilación mecánica (RM: 2.3; IC 95\%: 1.2-4.1) y bajo peso al nacimiento (RM: 2.6; IC 95\%: 1.0-6.8) $)^{11}$. El hemocultivo es el estándar de oro para el diagnóstico de sepsis neonatal, sin embargo, el índice de positividad es habitualmente bajo, siendo recomendable la toma de hemocultivos seriados, dado que en el tercer cultivo la positividad se presenta en un $99 \%{ }^{12}$. El control de infecciones eficaz requiere un cumplimiento completo de todo el personal con zonas de detección, prevención y control incluyendo precauciones estándar y de aislamiento, higiene de manos, cuidados de piel y equipo invasivo, vigilancia de antimicrobianos y compromiso de la atención centrada en la familia ${ }^{12}$. La Organización Mundial de la Salud y Centers for Disease Control and Prevention, recomiendan de manera enfática los geles para manos sin agua, a base de alcohol. Estos productos antisépticos previenen la transmisión de microorganismos patógenos más eficazmente que el lavado estándar o la antisepsia de las manos con jabones antimicrobianos ${ }^{12}$. El tratamiento antimicrobiano de los recién nacidos con sospecha de sepsis debe iniciarse oportunamente, como los signos de sepsis neonatal temprana no son específicos y el tratamiento inmediato con antibióticos ha demostrado reducir la mortalidad, una gran población de recién nacidos reciben tratamiento empírico basado en los factores de riesgo. El 95\% de los recién nacidos ingresados en la unidad de cuidados intensivos neonatales recibe tratamiento antibiótico empírico durante los primeros días de vida, a pesar de que los hemocultivos positivos para bacterias son del $1.5 \%$. El éxito del tratamiento empírico requiere del reconocimiento precoz de la infección, de una terapia antimicrobiana apropiada y de un soporte respiratorio, quirúrgico y cardiovascular dinámico. A fin de orientar la prescripción empírica, es crucial controlar los cambios en el patrón de organismos causales y su sensibilidad a los antimicrobianos. La evidencia científica sobre qué tipo de tratamiento debe ser preferido es pobre; el fármaco más 
frecuentemente utilizado es la ampicilina, seguida de gentamicina y cefotaxima. Se ha recomendado generalmente 14 días de tratamiento antimicrobiano en sepsis comprobada. La justificación y la seguridad de estos nunca han sido evaluadas científicamente. La duración del tratamiento se ha establecido clásicamente en 10 a 14 días para la sepsis sin infección focal ${ }^{13}$.

\section{Material y métodos}

Se realizó un estudio descriptivo, transversal, retrospectivo y observacional. Se revisaron todos los expedientes de los pacientes ingresados a la Unidad de Cuidados Intensivos Neonatales Internos y Externos del Hospital Materno Perinatal Mónica Pretelini Sáenz desde enero de 2013 hasta diciembre de 2015, en los cuales se tuviera el diagnóstico de sepsis neonatal con germen aislado en hemocultivo central.

\section{Resultados}

Con el propósito de dar a conocer los factores relacionados con sepsis neonatal en una unidad de cuidados intensivos neonatales, en el Hospital Materno Perinatal Mónica Pretelini Sáenz, en el cual hay un aproximado de 12,000 nacimientos anuales, y de los cuales se ingresan 4,500 por diversos motivos, se incluyeron para la realización de este estudio un total de 378 expedientes de recién nacidos que nacieron en este hospital en el periodo comprendido entre enero de 2013 y diciembre de 2015, en los cuales se encontró el diagnóstico de sepsis neonatal con germen aislado mediante hemocultivo central, 123 expedientes en el año 2013, 128 expedientes en 2014 y 127 expedientes en 2015.

En relación con el sexo del recién nacido más frecuentemente encontrado en estos tres años, fue el femenino, con un total de 209 expedientes, lo que constituye el $55.4 \%$, seguido por el masculino con un total de 175 expedientes (46.2\%). Los principales diagnósticos de ingreso, aunado a sepsis neonatal son bajo peso para la edad gestacional, así como prematurez, motivo por el cual se encontró un porcentaje importante de prematuros entre 29 y 34 semanas de gestación $(46 \%)$ y un peso mayor al ingreso de 1,500 gramos (67\%) (Tabla 1).

En cuanto al uso de procedimientos invasivos como colocación de tubo endotraqueal para uso de ventilación mecánica asistida, se encontraron un total de 260 expedientes de recién nacidos en los cuales se utilizó ventilación mecánica, lo que constituye un $68.8 \%$.
Tabla 1. Características antropométricas de la población*

\begin{tabular}{|c|c|c|}
\hline \multicolumn{3}{|c|}{ Pacientes con sepsis ( $n=378$ ) } \\
\hline Sexo femenino & 209 & $55.4 \%$ \\
\hline Sexo masculino & 169 & $44.6 \%$ \\
\hline SDG & 34.1 & $\pm 3.4 \mathrm{DE}$ \\
\hline Peso $<1,000 \mathrm{~g}$ & $943.6 \mathrm{~g}$ & $\pm 58.2 \mathrm{DE}$ \\
\hline Peso $1,001-1,500 \mathrm{~g}$ & $1,366.3 \mathrm{~g}$ & $\pm 141.8 \mathrm{DE}$ \\
\hline Peso $>1,501 \mathrm{~g}$ & $2,200 \mathrm{~g}$ & $\pm 549 \mathrm{DE}$ \\
\hline
\end{tabular}

*Las variables con distribución normal son dadas como media y desviación. Las medidas de frecuencia están dadas como porcentaje.

SDG: semanas de gestación; DE: desviación estándar.

Fuente: expedientes clínicos del Hospital Materno Perinatal Mónica Pretelini Sáenz.

El tiempo promedio en el cual se utilizó ventilación mecánica con uso de cánula orotraqueal, descartando toda aquella ventilación no invasiva (presión positiva continua de las vías respiratorias nasal, puntas nasales, oxígeno indirecto) se usó con un promedio de nueve días.

El uso de nutrición parenteral se encontró documentado en 283 expedientes, lo que constituye el $74.9 \%$, y solo el $26 \%$ no la utilizó. En promedio se utilizó por nueve días.

El uso de antiácidos que se documentó, dentro de los que agrupamos inhibidores $\mathrm{H} 2$ (ranitidina) e inhibidores de la bomba de protones (omeprazol), fue un $56.5 \%$, con un total de 215 expedientes, y 165 (43.5\%) no los utilizó.

En cuanto a la presencia de ruptura de membranas mayor a 18 horas como factor relacionado a sepsis neonatal se encontró que un $39.2 \%$ de los expedientes que se revisaron tenían este antecedente, no encontrando una fuerte asociación con esta variable.

La presencia de corioamnionitis materna documentada en el expediente del recién nacido bajo criterios estandarizados se encontró presente en un $59.5 \%$ del total revisado con 225 expedientes.

Estos dos últimos factores asociados han sido ampliamente estudiados, y en el total de expedientes revisados se encontró que en un $25 \%$ del total de la muestra se encuentra presente tanto la ruptura de membranas mayor a 18 horas como corioamnionitis materna (Tabla 2).

Los microorganismos involucrados más frecuentemente son de etiología bacteriana. En esta unidad el microorganismo aislado con mayor porcentaje fue E. coli, con un total de 84 casos reportados, lo que constituye un $22.3 \%$, seguido de $K$. pneumoniae con 


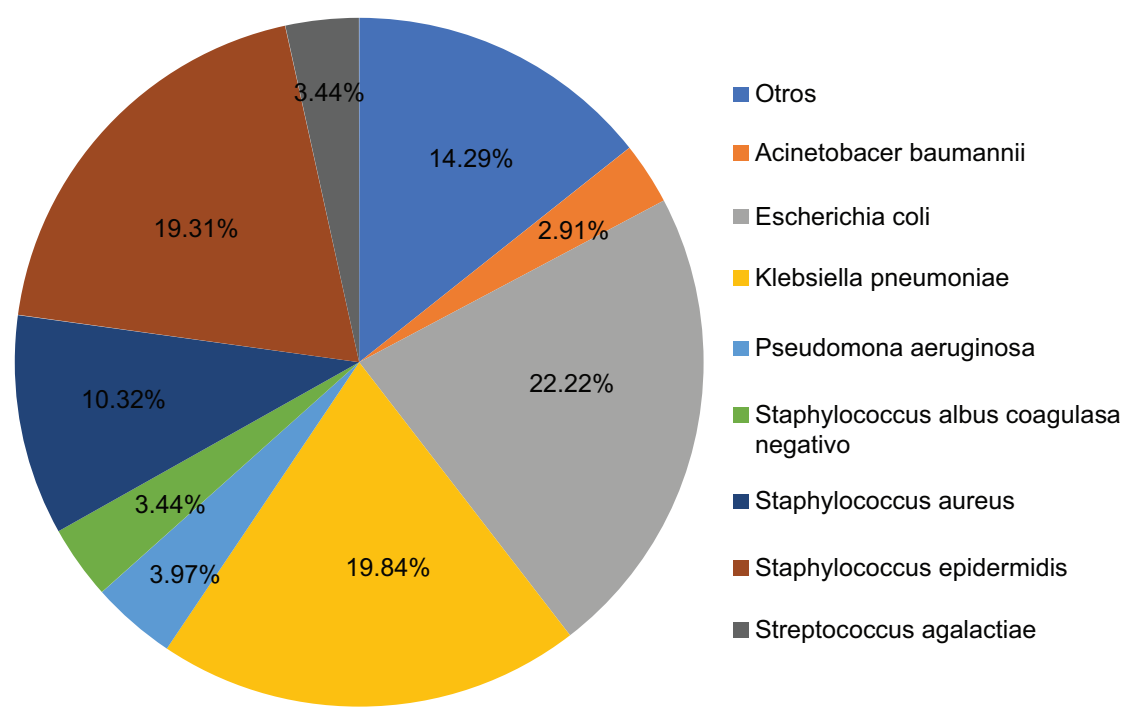

Figura 1. Tipo de gérmenes en neonatos con sepsis.

Tabla 2. Factores relacionados con sepsis neonatal*

\begin{tabular}{|c|c|c|}
\hline Corioamnionitis materna & 225 & $59.5 \%$ \\
\hline $\begin{array}{l}\text { Ruptura prolongada de membranas }>18 \\
\text { horas }\end{array}$ & 148 & $39.2 \%$ \\
\hline Uso de antiácidos & 161 & $42.8 \%$ \\
\hline Días de hospitalización & 23 & $14-39$ \\
\hline VM & 260 & $68.8 \%$ \\
\hline Días VM & 9 & $5-15$ \\
\hline CVC & 378 & $100 \%$ \\
\hline Días CVC & 18 & $12-28$ \\
\hline NPT & 283 & $74.9 \%$ \\
\hline Días de NPT & 9 & $4-15$ \\
\hline
\end{tabular}

*Las variables con distribución libre son dadas como mediana y p25-p75. Las medidas de frecuencia están dadas como porcentaje.

NPT: nutrición parenteral total; CVC: catéter venoso central; VM: ventilación mecánica.

Fuente: expedientes clínicos del Hospital Materno Perinatal Mónica Pretelini Sáenz.

un total de 75 casos reportados, constituyendo el $19.9 \%$, y en tercer lugar encontramos a Staphylococcus epidermidis, con un total de 73 casos $(19.9 \%)$ (Fig. 1).

Dentro de los microorganismos aislados con mayor frecuencia en sepsis temprana se encontró Staphylococcus albus coagulasa negativo, seguido de $S$. aureus, E. coli y $S$. epidermidis.
Los agentes causales en sepsis tardía más frecuentemente encontrados fueron $S$. aureus, seguido de K. pneumoniae y Pseudomona aeruginosa (Fig. 2).

El uso de accesos vasculares centrales se usó en el $100 \%$ de los expedientes revisados, sin embargo, cabe resaltar que dentro del total entran el uso de onfaloclisis, catéter percutáneo periférico y catéter arrow; se vio que el número promedio de días en el cual se utilizó esta vía central fue de 18. La presencia de sepsis tardía se encontró en un $45 \%$, con un total de 170 expedientes, y sepsis temprana en 208 expedientes (55\%). Hicimos un grupo de sepsis temprana y sepsis tardía, en el cual comparamos cada una de las variables en estudio. En sepsis temprana $(n=208)$ encontramos que el sexo femenino se encontró en 117 (56\%), con una edad gestacional de $34.14 \pm 0.23$ y corioamnionitis materna en 129 (57.3\%) (Tabla 3). Las variables se encuentran agrupadas de acuerdo con el tipo de sepsis que se encontró (temprana o tardía), en las cuales se observó una diferencia entre ambos grupos.

Se realizó un modelo de regresión lineal, para poder conocer el riesgo de cada una de las variables o factores de riesgo que pueden modificar el desenlace, donde se realiza un análisis bivariante solo utilizando las variables que hayan alcanzado una $p<0.20$. En estas se buscó una odds ratio (OR), con un IC 95\%, donde aquellos valores con un IC en 1 no son significativos y que se corrobore con una $p>0.05$ o no significativa (Tabla 4). 


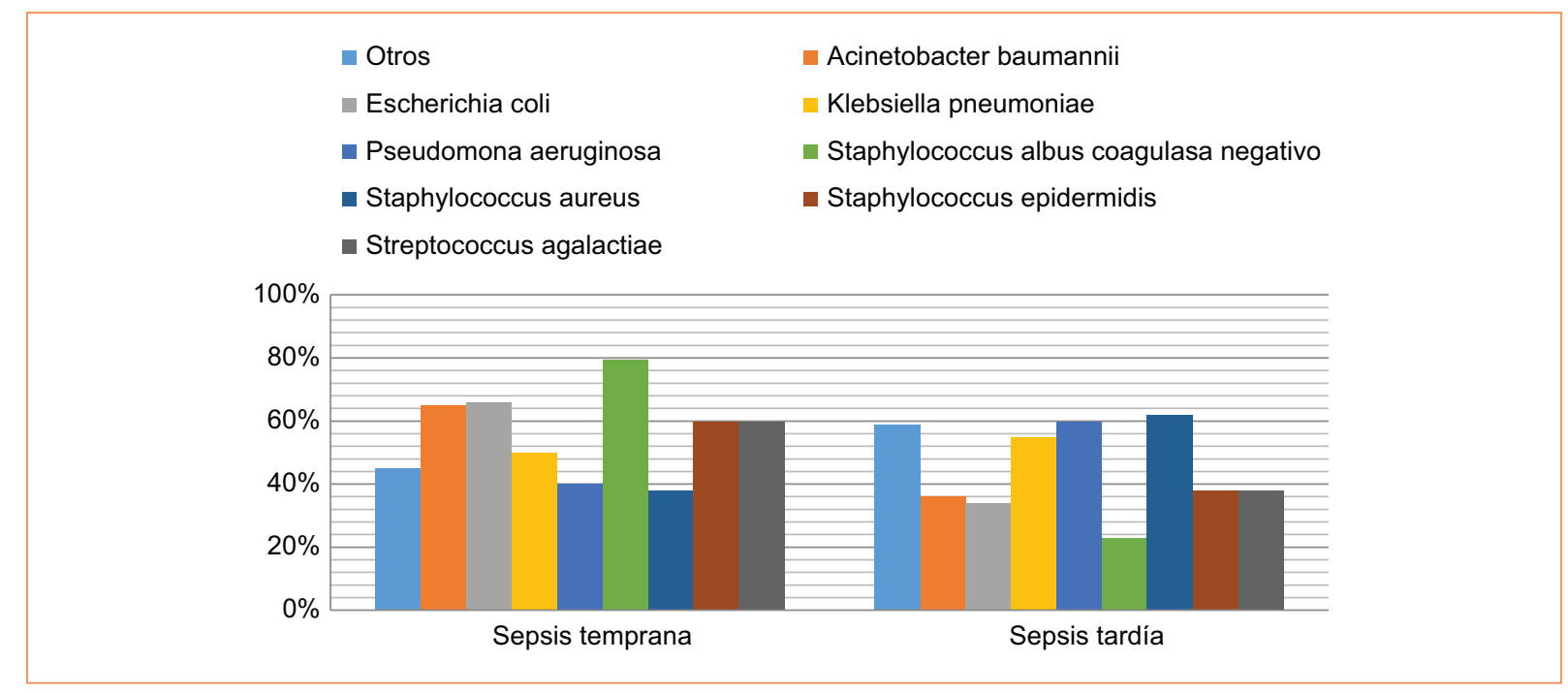

Figura 2. Tipo de gérmenes por grupo.

Tabla 3. Comparación de variables sepsis temprana y tardía*

\begin{tabular}{|c|c|c|c|}
\hline & $\begin{array}{l}\text { Sepsis temprana } \\
\quad(\mathrm{n}=\mathbf{2 0 8})\end{array}$ & $\begin{array}{l}\text { Sepsis tardía } \\
\quad(\mathbf{n}=170)\end{array}$ & $\mathbf{p}$ \\
\hline Sexo femenino $\mathrm{n}(\%)$ & $117(56)$ & $92(44)$ & 0.725 \\
\hline SDG & $34.14 \pm 0.23$ & $34.06 \pm 0.25$ & 0.813 \\
\hline Peso $<1,000 \mathrm{~g}$ & $941.3 \pm 11.4$ & $947.2 \pm 19$ & 0.779 \\
\hline Peso $1,001-1,500 \mathrm{~g}$ & $1,349.6 \pm 23.6$ & $1,379 \pm 19.2$ & 0.331 \\
\hline Peso $>1,501 \mathrm{~g}$ & $2,338.7 \pm 46.8$ & $2,267.8 \pm 55.9$ & 0.331 \\
\hline Corioamnionitis materna & $129(57.3)$ & $96(42.7)$ & 0.274 \\
\hline Ruptura prolongada de membranas $>18 \mathrm{~h}$ & $89(60.1)$ & 59 (39.9) & 0.109 \\
\hline Uso de antiácidos & $86(53.4)$ & $75(46.6)$ & 0.581 \\
\hline Días de hospitalización & $31.8 \pm 1.8$ & $32.4 \pm 2.1$ & 0.835 \\
\hline VM & $134(51.5)$ & $126(48.5)$ & 0.043 \\
\hline Días con uso de VM & $7.30 \pm 0.52$ & $8.2 \pm 0.57$ & 0.256 \\
\hline CVC & $208(55.2)$ & $169(44.8)$ & 0.268 \\
\hline Días con CVC & $21.8 \pm 0.98$ & $21.9 \pm 1.15$ & 0.969 \\
\hline NPT & $152(53.7)$ & $131(46.3)$ & 0.375 \\
\hline Días con NPT & $8.2 \pm 0.57$ & $8.4 \pm 0.58$ & 0.823 \\
\hline Muerte & $22(73.3)$ & $8(26.7)$ & 0.036 \\
\hline
\end{tabular}

*Las variables con distribución libre son dadas como mediana y p25-p75. Las medidas de frecuencia están dadas como porcentaje.

SDG: semanas de gestación; NPT: nutrición parenteral total; CVC: catéter venoso central; VM: ventilación mecánica.

Fuente: expedientes clínicos del Hospital Materno Perinatal Mónica Pretelini Sáenz.

La ruptura prolongada de membranas mayor a 18 horas se encontró en sepsis temprana en 89 expedientes $(60.1 \%)$ y en sepsis tardía en $59(39.9 \%)$, con una $p=0.109$, siendo no significativa. La OR con IC $95 \%$ fue de $1.39(0.911-2.14)$ con una $p=0.125$, continuando sin ser significativo. 
Tabla 4. Modelo con las variables en estudio

\begin{tabular}{|l|c|c|}
\hline Variable & OR (IC 95\%) & $P$ \\
\hline Peso al nacer & $1.0002(0.99-1.0006)$ & 0.89 \\
\hline $\mathrm{P}<1 \mathrm{~kg}$ & $1.613(0.71-3.65)$ & 0.251 \\
$\mathrm{P} 1-1.5 \mathrm{~kg}$ & $0.45(0.18-1.07)$ & 0.072 \\
$\mathrm{P}>1.5 \mathrm{~kg}$ & $0.83(0.35-1.97)$ & 0.679 \\
\hline Semanas de gestación & $0.975(0.90-1.056$ & 0.548 \\
\hline Corioamnionitis & $1.03(0.58-1.80)$ & 0.915 \\
\hline Ruptura prolongada de & $1.42(0.80-2.52)$ & 0.218 \\
\hline membranas & & \\
\hline Ventilación mecánica & $0.652(0.386-1.10)$ & 0.111 \\
\hline Sexo (femenino) & $0.959(0.630-1.46)$ & 0.847 \\
\hline Antiácidos & $1.11(0.695-1.79)$ & 0.46 \\
\hline
\end{tabular}

P: peso; OR: odds ratio; IC 95\%: intervalo de confianza del $95 \%$. Fuente: expedientes clínicos del Hospital Materno Perinatal Mónica Pretelini Sáenz.

El uso de antiácidos se encontró en sepsis temprana en 86 expedientes $(53.4 \%)$ y en sepsis tardía en $75(46.6 \%)$, con una $p=0.58$, lo cual tampoco es significativo, en ambos grupos.

La ventilación mecánica estuvo presente en sepsis temprana en 134 expedientes (51.5\%) y en sepsis tardía en 126 (48.5\%), con una $p=0.043$ (significativa). La OR con un IC $95 \%$ de 1.39 (0.911-2.14) con una $\mathrm{p}=0.125$ siendo significativo y fuertemente relacionado con sepsis neonatal.

La nutrición parenteral total estuvo presente en sepsis temprana en 152 expedientes (53.7\%) y en sepsis tardía en 131 (46.3\%), con una $p=0.375$, lo cual no es significativo. La mortalidad en sepsis temprana se presentó en el $22 \%$ de los expedientes revisados y un $8 \%$ en el grupo de sepsis tardía, con una $p=0.036$.

\section{Conclusiones}

La mayoría de los factores de riesgo identificados en nuestro estudio son modificables, por lo que la generación de estrategias permitirá disminuir la incidencia de sepsis con germen aislado en el recién nacido y el impacto económico que representa para nuestra institución, además de reducir las secuelas ocasionadas por esta patología en nuestra población en estudio. La prematurez y el bajo peso siguen siendo los factores de riesgo que obligan al uso de procedimientos invasivos en los recién nacidos.

\section{Financiamiento}

La presente investigación no ha recibido ninguna beca específica de agencias de los sectores públicos, comercial o con ánimo de lucro.

\section{Conflicto de intereses}

Los autores declaran no tener conflicto de intereses.

\section{Responsabilidades éticas}

Protección de personas y animales. Los autores declaran que para esta investigación no se han realizado experimentos en seres humanos ni en animales.

Confidencialidad de los datos. Los autores declaran que han seguido los protocolos de su centro de trabajo sobre la publicación de datos de pacientes.

Derecho a la privacidad y consentimiento informado. Los autores han obtenido el consentimiento informado de los pacientes y/o sujetos referidos en el artículo. Este documento obra en poder del autor de correspondencia.

\section{Bibliografía}

1. Disminuye la mortalidad neonatal, pero aumenta su proporción en la mortalidad en la niñez a escala mundial [Internet]. Organización de Naciones Unidas, Comisión Económica para América Latina y el Caribe; 30/agosto/2011. Disponible en: https://www.cepal.org/fr/node/ 42967

2. MacDonald MG, Seshia MK. Avery. Neonatología. Diagnóstico y tratamiento del recién nacido. 7.ㄹ edición. Wolters Kluwer; 2017.

3. Tijerina TC, Rodríguez BI. Sepsis neonatal intrahospitalaria. Incidencia y factores de riesgo. Rev Med Inst Mex Seguro Soc. 2011;49:643-8.

4. Fernández Colomer B, López Sastre J, Coto Cotallo GD, Ramos Aparicio A, Ibáñez Fernández A. Sepsis en el recién nacido. Protocolos diagnósticos terapéuticos de la Asociación Española de Pediatría [Internet]. Asociación Española de Pediatría; 2008. Disponible en: https://www. aeped.es/sites/default/files/documentos/21_0.pdf

5. Flores-Herrera H, Maida-Claros R, Solís-Herrera H, Illescas-Medrano E, Zavala-Díaz de la Serna FJ. Identificación molecular de bacterias causales de sepsis neonatal mediante la reacción en cadena de la polimerasa (PCR). Acta Pediatr Mex. 2009;30(3):148-55.

6. Venkatesh M, Flores A. Molecular microbiological methods in the diagnosis of neonatal sepsis. Expert Rev Anti Infect Ther. 2010;8(9):1037-48

7. Ecker DJ, Sampath R, Li H, Massire C, Matthews HE, Toleno D, et al. New technology for rapid molecular diagnosis of bloodstream infections. Expert Rev Mol Diagn. 2010;10(4):399-415.

8. Rhee V, Mullany LC, Khatry SK, Katz J, LeClerq SC. Maternal and birth attendant hand washing and neonatal mortality in southerm Nepal. Arch Pediatric Adolesc Med. 2008;162:603-8.

9. Thaver D, Zaidi AK. Burden of neonatal infections in developing countries: a review of evidence from community-based studies. Pediatr Infect Dis J. 2009;28(1 Suppl):S3-S9.

10. Xiao T, Chen LP, Liu H, Xie S, Luo Y, Wu DC. The analysis of etiology and risk factors for 192 case of neonatal sepsis. Biomed Res Int. 2017;2017:8617076.

11. Bentlin MR, Rugolo LM. Late-onset sepsis: Epidemiology, evaluation and outcome. NeoReviews. 2010;11(8);426-35.

12. Stoll BJ, Hansen N. Early onset neonatal sepsis: the burden of group $B$ Streptococcal and E. coli disease continues. Pediatrics. 2011;127(5): 817-26.

13. Gathwala G, Sindwani A, Singh J, Choudhry U. Ten days vs 14 days antibiotic therapy in culture-proven neonatal sepsis. J Trop Pediatrics. 2010;56(6):2010. 